\title{
Leukocyte Count versus C-Reactive Protein Levels in Obese Portuguese Patients Aged 6-12 Years Old
}

\author{
Henrique Nascimento*, ${ }^{1,2}$, Susana Rocha ${ }^{1,2}$, Carla Rego ${ }^{3}$, Helena Ferreira Mansilha ${ }^{4}$, Alexandre \\ Quintanilha $^{5}$, Alice Santos-Silva ${ }^{1,2}$ and Luís Belo* ${ }^{* 1,2}$ \\ ${ }^{I}$ Department of Biochemistry, Faculty of Pharmacy, University of Porto, 4050-047 Porto, Portugal \\ ${ }^{2}$ Institute for Molecular and Cell Biology (IBMC), University of Porto, 4150-180 Porto, Portugal \\ ${ }^{3}$ Nutrition Unit / Paediatric Service. UAG-MC. Hospital de S. João E.P.E. Faculty of Medicine of Porto University; \\ 4200-319 Porto, Portugal \\ ${ }^{4}$ Paediatric Department of Maria Pia Children's Hospital; 4050-111, Porto, Portugal \\ ${ }^{5}$ Institute of Biomedical Sciences of Abel Salazar (ICBAS), University of Porto, 4099-003 Porto, Portugal
}

\begin{abstract}
Objectives: to evaluate whether total and differential WBC counts are altered in young obese patients (aged 612 years) and if a relationship exists between WBC counts and the severity of obesity as well as with CRP level.

Materials and Methods: a group of 77 obese patients [32 males and 45 females] and 19 controls [7 males and 12 females] were studied. Total WBC count was performed by using an automatic blood cell counter. Blood cell morphology and WBC differential count were evaluated in Wright stained blood films. The plasma levels of CRP were evaluated by immunoturbidimetry.

Results: obese participants presented with a statistically significant higher neutrophil percentage and CRP levels when compared to controls; the median CRP value was about 5 times higher than that observed in controls. Absolute neutrophil count and neutrophil/lymphocyte ratio were also higher in patients, though without statistical significance. The parameters that were statistically significant related with adiposity markers were neutrophil count and CRP levels. The neutrophil count was positively and statistically correlated with body mass index (BMI), BMI z-score, waist circumference and waist/height ratio, and also with CRP levels. In multiple regression analysis, the only variable that remained statistically associated with neutrophil count was CRP (neutrophil count $=2.612+0.4391$ CRP; standardised coefficient/beta: 0.384 , $P=0.001$ ). When performing multiple regression without CRP, the only variable that remained statistically associated with neutrophil count was BMI.

Conclusions: our results demonstrated in obese patients aged 6-12 years, a significant change in the differential leukocyte count towards neutrophilia, together with a significant higher CRP concentration, and that absolute neutrophil count correlates with obesity markers and with CRP levels. Our data also indicate that neutrophil count, a current clinically used low-cost parameter, may be used as an obesity-related inflammatory marker in young obese patients.
\end{abstract}

Keywords: Leukocytes, C-reactive protein, children obesity.

\section{INTRODUCTION}

Obesity is increasing all over the world, particularly in industrialised countries, and is a significant risk factor for cardiovascular disease (CVD). This is of particular concern in our country, as demonstrated by the high prevalence of overweight/obesity $(31.5 \%)$ in Portuguese children, compared to other European countries [1]. Furthermore, Portugal exhibits a high rate of CVD, particularly of cerebrovascular disease.

\footnotetext{
*Address correspondence to these authors at the Departamento de Bioquímica, Faculdade de Farmácia, Universidade do Porto, 4050-047 Porto, Portugal; Tel: +351-222078906; Fax: +351-222003977;

E-mail: henriqueferrao@hotmail.com

Departamento de Bioquímica, Faculdade de Farmácia, Universidade do Porto, 4050-047 Porto, Portugal; Tel: +351-222078906; Fax: +351222003977; E-mail: luisbelo@ff.up.pt
}

Cardiovascular morbidity and mortality of obesity is associated with classic risk factors, namely dyslipidemia, hypertension and impaired glucose metabolism [2, 3]. Recently, it was shown that obesity may be regarded as a state of low-grade inflammation [3,4] and atherosclerosis is accepted as an inflammatory process [5]. The adipocyte is an important source of cytokines, namely interleukin (IL)-6 and tumour necrosis factor (TNF)-alpha, and their levels are significantly higher in the plasma of obese patients [4, 6]. The rise in these cytokines, especially in IL-6, triggers an increased synthesis of C-reactive protein (CRP), one of the most sensitive makers of inflammation [7]. However, and although obesity is recognized as a possible cause for reactive leukocytosis [8], the contribution of leukocytes to the inflammatory process in obese patients is not fully understood. 
To date, limited investigations have examined the associations between white blood cell (WBC) count and obesity in children and adolescents. A National Health and Nutrition Examination Survey of the US population demonstrated that overweight is associated with higher WBC count in children [9]. Furthermore, a recent study performed in female obese adolescents showed that WBC count is positively related to body mass index (BMI), waist circumference, and total adipose tissue (TAT) and subcutaneous adipose tissue (SAT) areas [10]. After adjustment for metabolic risk factors, only neutrophil counts were positively related to BMI, waist circumference, and TAT; lymphocyte and basophil counts were negatively related to BMI and waist circumference [10]. This study was performed only in female adolescents (aged 10-19 years) and no control group was used.

It is still uncertain which WBC subfractions are more altered in obese children and adolescents, and how prematurely these changes occur in obesity. Thus, the aim of our work was to evaluate whether total and differential WBC counts are altered in young obese patients (aged 6-12 years) and if a relationship exists between WBC counts and the severity of obesity (according to BMI z-score, waist circumference and waist/height ratio) as well as with CRP level, a known sensitive marker of inflammation.

\section{MATERIAL AND METHODS}

\subsection{Subjects}

The protocol used for all participants was approved by the Committees on Ethics of the University Hospital S. João and of the Children's Hospital Maria Pia, Porto. Obese patients, aged 6-12 years, were identified from medical records at the Departments of Pediatrics of Hospital Maria Pia and Hospital S. João, Porto. All children that reached inclusion criteria were invited to participate in the study. Seventy seven obese children participated in the study after informed and written consent of their parents. The study took place between May 2006 and March 2007.

Healthy control subjects, age and sex matched with obese patients, were recruited from the general population.

Obesity was defined as BMI greater than the 95th percentile for age and gender, according to 2000 Centre for Disease Control and Prevention (CDC) growth charts. Because BMI is not normally distributed, we used bodymass index z-score (BMI $\mathrm{z}$-sc); BMI $\mathrm{z}$-sc values were achieved by using a calculator that has per basis the 2000 CDC growth charts.

Clinical data regarding the sample population was collected. The physical examination included the measurement of height, weight, waist circumference, and the presence of skin lesions related with obesity and its comorbidities.

The participants were invited to the research centers after an overnight fast and, after clinical examination, blood was collected for laboratory analysis.

Smokers, subjects with diabetes mellitus, endocrine disorders, hereditary diseases, inflammatory or infectious diseases or under any therapy that could interfere with our results were excluded from the study.

\subsection{Procedures and Assays}

\subsubsection{Blood Samples}

Fasted blood samples were obtained and processed within $2 \mathrm{~h}$ of collection. Blood was obtained by venipuncture in EDTA containing tubes. Plasma aliquots were made and immediately stored at $-70^{\circ} \mathrm{C}$ until assayed.

\subsubsection{Laboratory Analysis}

Total WBC count was performed by using an automatic blood cell counter (ABX Micros 60-OT). Blood cell morphology and WBC differential count were evaluated in Wright stained blood films. The plasma levels of CRP were evaluated by immunoturbidimetry [CRP (latex) HighSensitivity, Roche Diagnostics].

\subsection{Statistical Analysis}

Statistic analysis was performed using the Statistical Package for Social Sciences (SPSS, version 17.0) for Windows. Kolmogorov-Smirnov analysis was used to test if the results were normally distributed. The results normally distributed are presented as mean \pm SD and those notnormally distributed are presented as median (interquartile range).

Controls and obese patients were compared using Student's unpaired $t$ test or Mann-Whitney $U$ test. The distribution of males and females with respect to categorical variables was analysed using chi-squared $\left(\chi^{2}\right)$ test and Fisher's exact test.

The strength of the association between the substances was estimated by Pearson correlation coefficient, after logarithmic transformation of the variables (when necessary). To evaluate the contribution of the different variables to neutrophil count, we performed multiple regression analysis, using stepwise selection, with an entry criteria of $P<0.05$. Significance was accepted at $P$ less than 0.05 .

\section{RESULTS}

The demographic and clinical characteristics of the obese participants $(n=77)$ and controls $(n=19)$ are presented in Table 1. The groups were matched for age and gender; as expected, BMI and BMI z-sc values were significantly higher in patients.

Obese participants presented with a statistically significant higher neutrophil percentage and CRP levels (Table 2). Absolute neutrophil count and neutrophil/1 ymphocyte ratio were also higher in patients, though without statistical significance.

Concerning the comparison between boys and girls, no statistical significant differences were observed within both groups (data not shown).

The correlations between CRP levels and the absolute count for each type of leukocyte with adiposity markers are presented in Table 3. The only parameters that were statistically significant related with adiposity markers were neutrophil count and CRP levels. The neutrophil count was positively and statistically correlated with BMI, BMI z-sc, waist circumference and waist/height ratio, and also with CRP levels. In the multiple regression analysis, the only 
Table 1. Characteristics of the Studied Subjects

\begin{tabular}{|l|c|c|c|}
\hline & $\begin{array}{c}\text { Controls } \\
(\boldsymbol{n}=\mathbf{1 9})\end{array}$ & $\begin{array}{c}\text { Obese patients } \\
(\boldsymbol{n}=\mathbf{7 7})\end{array}$ & $\boldsymbol{P}$ \\
\hline \hline Age (years) & $8.5 \pm 1.6$ & $9.1 \pm 1.6$ & 0.133 \\
\hline Gender male $(\boldsymbol{n})$ & $7(36.8 \%)$ & $27(41.6 \%)$ & 0.708 \\
\hline BMI $\left(\mathbf{k g} / \mathbf{m}^{2}\right)$ & $16.27 \pm 1.54$ & $2.331 \pm 0.317$ & $<0.001$ \\
\hline BMI z-sc & $0.005 \pm 0.820$ & $86.9 \pm 10.2$ & - \\
\hline Waist circumference $(\mathbf{c m})$ & - & $0.609 \pm 0.054$ & - \\
\hline Waist/Height ratio & - & & \\
\hline
\end{tabular}

Values are given as mean $\pm \mathrm{SD}$, unless otherwise indicated.

Table 2. Total and Differential Leukocyte Counts and CRP Levels in Controls and Obese Patients Aged 6-12 Years

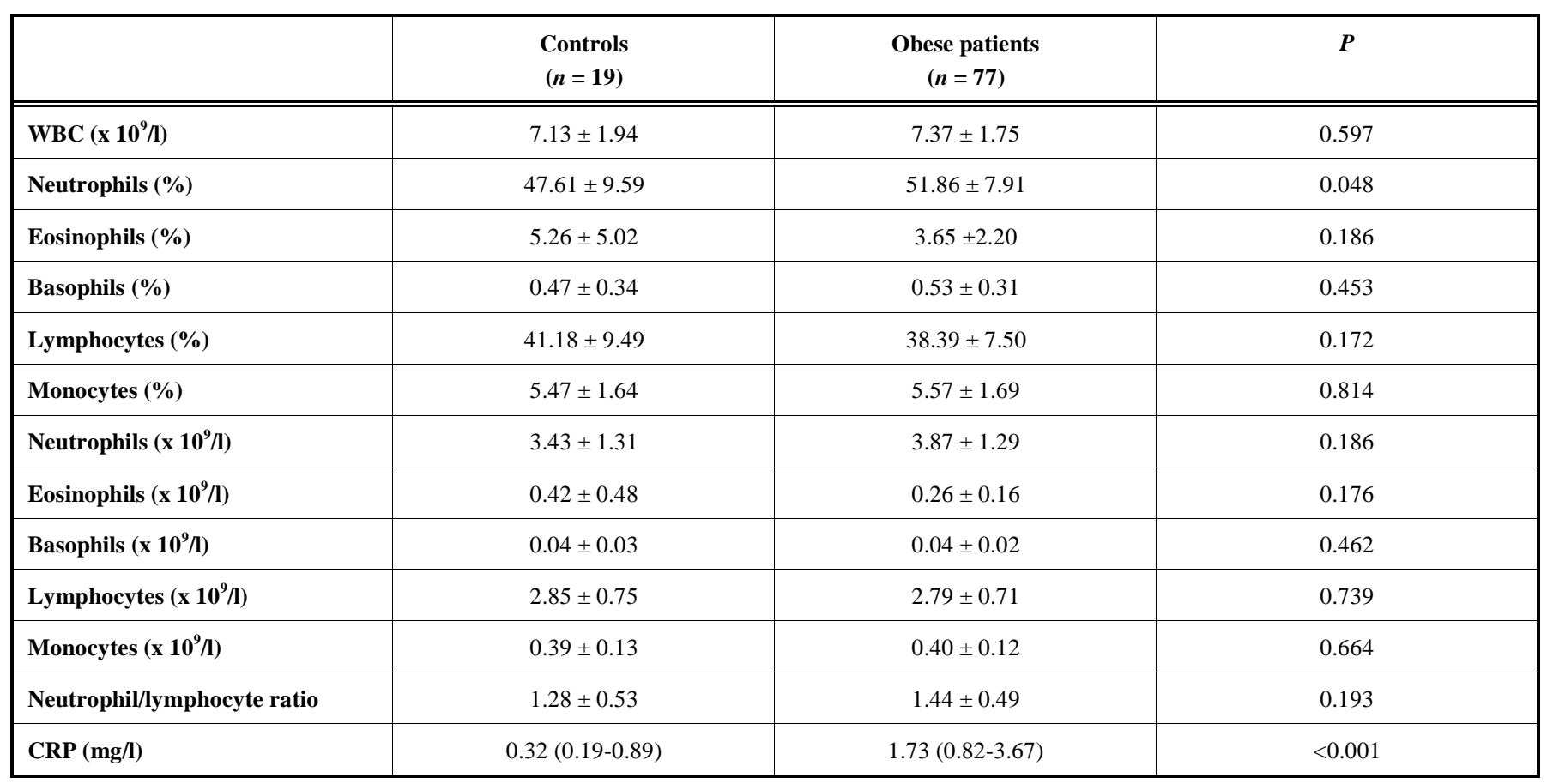

Values are given as mean \pm SD or median (interquartile range).

Table 3. Correlations Between CRP Level and the Absolute Count for Each Type of Leukocytes with Adiposity Markers, in Obese Patients Aged 6-12 Years

\begin{tabular}{|c|c|c|c|c|c|c|}
\hline & Neutrophils $\left(\times 10^{9} / \mathrm{l}\right)$ & Lymphocytes (x109/1) & Monocytes (x10 9 /l) & Eosiniphols (x10 $\left.{ }^{9} .1\right)$ & Basophils (x109.1) & Ln PCR \\
\hline BMI z-sc & $0.249^{*}$ & 0.120 & 0.058 & 0.064 & 0.113 & $0.313^{* *}$ \\
\hline $\begin{array}{l}\text { Waist } \\
\text { circumference (cm) }\end{array}$ & $0.225^{*}$ & 0.089 & 0.101 & 0.019 & -0.017 & 0.188 \\
\hline Ln CRP & $0.384 * *$ & 0.075 & $0.295^{*}$ & 0.014 & 0.051 & - \\
\hline
\end{tabular}

$* P<0.05 ; * * P<0.01$

variable that remained statistically associated with neutrophil count was CRP (neutrophil count $=2.612+0.4391$ nCRP; standardised coefficient/beta: $0.384, P=0.001$ ); BMI was excluded from this model but its partial correlation almost reached statistical significance $(0.212, P=0.067)$. When performing multiple regression without CRP, the only 
variable that remained statistically associated with neutrophil count was BMI (neutrophil count $=1.117+0.100 \mathrm{BMI}$; standardised coefficient/beta: $0.289, P=0.011$ ).

\section{DISCUSSION}

Leukocytosis is often associated with atherosclerotic disease and is accepted as a risk factor for CVD [11-13]. The association between leukocyte count and risk of atherosclerotic disease is plausible because leukocytes give a major contribution to the rheologic properties of blood, alter their own adhesive properties under stress and participate also in endothelial injury [11]. Moreover, the recruitment of monocytes and lymphocytes to the artery wall is characteristic of atherosclerosis [14].

Atherosclerosis is a chronic disease that begins early in life. Obesity is an important risk factor for the development of CVD, but, to date, limited investigations have examined the associations between WBC count and obesity in children and adolescents.

A study on subjects who were referred for further evaluation of leukocytosis by their family physicians demonstrated that obesity is the second most common cause of leukocytosis, being smoking the commonest cause [8]. Furthermore, in a previous large cross-sectional epidemiological study, performed in adults, an increased BMI was a statistically significant independent predictor of a higher peripheral blood leukocyte count [15]. Leptin, IL-1, IL- 6 and TNF- $\alpha$, all produced by the human adipose tissue, have been implicated in this leukocyte rise [8, 14, 16, 17]. However, as far as we know, this is the first study assessing the association of leukocyte count with obesity and its associated inflammation, assessed by CRP levels, in obese patients and (lean) controls aged 6-12 years.

As leukocyte count changes with age and is influenced by gender [15], we matched controls and patients for gender and age. We chose participants aged 6-12 years, as in this age range it is not normally observable significant changes in leukocyte counts. In our study, we also did not find any significant correlation between total and differential leukocyte counts with age.

The median CRP value was about 5 times higher than that observed in controls, demonstrating an inflammatory process in such young obese patients (Table 2). The total leukocyte count did not show a statistically significant change in obesity, but a different ratio between the different leukocyte types was observed, as showed by the significantly higher value in the percentage of neutrophils with obesity. Even though no significant differences in absolute neutrophil count were observed, patients presented a trend to higher values. Moreover, absolute neutrophil count correlated not only with markers of obesity but also with CRP levels. This suggests that neutrophil count may be used as a marker of obesity severity, with its associated inflammatory state. The fact that CRP levels remained statistically associated with neutrophil count by multiple regression analysis may be explained by the fact that there are cytokines, produced and released by the adipocyte, namely IL-6 and IL-1, that are able to induce both an increase in CRP synthesis and neutrophilia via demargination of neutrophils from the marginal "pool", acceleration of bone marrow neutrophil release or enhancement of bone marrow granulopoiesis [7, $18,19]$. It is worthy to emphasise that in our study, when CRP was removed from the model, BMI was the only variable that remained statistically associated with neutrophil count.

The significant positive correlations that we found between neutrophil count and BMI and waist circumference values are in agreement with those obtained by Kim and Park [10] in female obese adolescents aged 10-19 years. However, we were unable to find any correlation with absolute lymphocyte count. Kim and Park [10] also found that WBC count is strongly related to subcutaneous rather than visceral adiposity.

There are a lot of beneficial effects on health resulting from moderated aerobic physical exercise, namely a reduction in obesity markers (e.g. BMI) and in risk of type 2 diabetes, and an improvement in endothelial function and lipid profile, all risk factors for CVD [20-23]. Data from large study populations also suggest an inverse association between chronic physical activity and the level of some inflammatory markers (e.g. CRP and interleukin-6) [21, 24, 25]. In obese adult subjects who lost weight significantly, leukocytosis and the acute-phase reactants seem to gradually return to normal [8]. However, few data exists in literature regarding young obese subjects. It was shown that 3 months of moderate lifestyle intervention in obese adolescents seems to attenuate the inflammatory state associated with obesity, as observed by a reduction in elevated circulating concentrations of CRP, fibrinogen and IL-6, non-traditional risk factors for CVD [16]. However, and despite the consistency of the results, the small sample size of this study ( $n=8$ in the intervention group) calls for attention in strengthening these results with further studies. Reinehr et al. [26], by evaluating 16 obese children who lost weight over a 1-year period, found a significant decrease in CRP but no significant changes in TNF-alpha levels. If future research supports the idea that, at young ages, the protection provided by regular physical activity against obesity-mediated inflammation is relevant, this would encourage even more its practice. Moreover, at these young ages, this would also be a good opportunity to implement healthy lifestyles.

In conclusion, our results demonstrated a significant change in the differential leukocyte count towards neutrophilia, together with a significant higher CRP concentration, in obese patients aged 6-12 years, and that absolute neutrophil count correlates with obesity markers, especially BMI, and with CRP levels. Our data also indicate that neutrophil count, a current clinically used low-cost parameter, may be used as an obesity-related inflammatory marker in young obese patients. However, more studies are needed to confirm our findings, involving larger number of cases. Furthermore, young obese patients may deserve from physical activity programs.

\section{ACKNOWLEDGEMENTS}

The authors wish to thank the technicians Amélia Ferreira, Andreia Sousa, Joana Barros and Isabel Almeida for expert assistance on blood collection, and to the University of Porto (IPG07/2007) for financial support. 


\section{REFERENCES}

[1] Padez, C.; Fernandes, T.; Mourão, I.; Moreira, P.; Rosado, V. Prevalence of overweight and obesity in 7-9-year-old Portuguese children: trends in body mass index from 1970-2002. Am. J. Hum. Biol., 2004, 16(6), 670-8.

[2] Alexander, C.M.; Landsman, P.B.; Grundy, S.M. The influence of age and body mass index on the metabolic syndrome and its components. Diabetes Obes. Metab., 2008, 10(3), 246-50.

[3] Shah, A.; Mehta, N.; Reilly, M.P. Adipose inflammation, insulin resistance, and cardiovascular disease. JPEN J. Parenter. Enteral. Nutr., 2008, 32(6), 638-44.

[4] Berg, A.H.; Scherer, P.E. Adipose tissue, inflammation, and cardiovascular disease. Circ. Res. 2005, 96(9), 939-49.

[5] Ross, R. Atherosclerosis - an inflammatory disease. N. Engl. J. Med. 1999, 340(2), 115-26.

[6] Rondinone CM. Adipocyte-derived hormones, cytokines, and mediators. Endocrine, 2006, 29(1), 81-90.

[7] Kushner, I.; Rzewnicki, D. Acute phase response. In: Gallin JI, Snyderman R, Fearon DT, Haynes BF, Nathan C, Eds. Inflammation: Basic Principles and Clinical Correlates. $3^{\text {rd }}$ ed. Philadelphia: Lippincott Williams \& Wilkins; 1999, pp. 317-29.

[8] Herishanu, Y.; Rogowski, O.; Polliack, A.; Marilus, R. Leukocytosis in obese individuals: possible link in patients with unexplained persistent neutrophilia. Eur. J. Haematol., 2006, 76, 516-20.

[9] Visser, M.; Bouter, L.M.; McQuillan, G.M.; Wener, M.H.; Harris, T.B. Low-grade systemic inflammation in overweight children. Pediatrics, 2001, 107(1), E13.

[10] Kim, J.A.; Park, H.S. White blood cell count and abdominal fat distribution in female obese adolescents. Metabolism, 2008, 57(10), 1375-9.

[11] Ernst, E.; Hammerschmidt, D.E.; Bagge, U.; Matrai, A.; Dormandy, J.A. Leukocytes and the risk of ischemic diseases. JAMA, 1987, 257(17), 2318-24.

[12] Danesh, J.; Collins, R.; Appleby, P.; Peto, R. Association of fibrinogen, C-reactive protein, albumin, or leukocyte count with coronary heart disease: meta-analyses of prospective studies. JAMA, 1998, 279(18), 1477-82.

[13] Danesh, J.; Whincup, P.; Walker, M.; Lennon, L.; Thomson, A.; Appleby, P.; Gallimore, J.R.; Pepys, M.B. Low grade inflammation and coronary heart disease: prospective study and updated metaanalyses. BMJ, 2000, 321(7255), 199-204.

[14] Lusis, A.J. Atherosclerosis. Nature, 2000, 407(6801), 233-41.

[15] Schwartz, J.; Weiss, S.T. Host and environmental factors influencing the peripheral blood leukocyte count. Am. J. Epidemiol., 1991, 134(12), 1402-1409.
[16] Balagopal, P.; George, D.; Patton, N.; Yarandi, H.; Roberts, W.L.; Bayne, E.; Gidding S. Lifestyle-only intervention attenuates the inflammatory state associated with obesity: a randomized controlled study in adolescents. J. Pediatr., 2005, 146(3), 342-8.

[17] Stewart, R.A.; White, H.D.; Kirby, A.C.; Heritier, S.R.; Simes, R.J.; Nestel, P.J.; West, M.J.; Colquhoun, D.M.; Tonkin, A.M.; Long-Term Intervention With Pravastatin in Ischemic Disease (LIPID) Study Investigators. White blood cell count predicts reduction in coronary heart disease mortality with pravastatin. Circulation, 2005, 111(14), 1756-62.

[18] Suwa, T.; Hogg, J.C.; English, D.; Van-Eeden, S.F. Interleukin-6 induces demargination of intravascular neutrophils and shortens their transit in marrow. Am. J. Physiol. Heart Circ. Physiol. 2000, 279(6), H2954-60.

[19] Veltri, S.; Smith-2nd J.W. Interleukin 1 trials in cancer patients: a review of the toxicity, antitumor and hematopoietic effects. Stem Cells, 1996, 14(2), 164-76.

[20] Tsai, A.C.; Sandretto, A.; Chung, Y.C. Dieting is more effective in reducing weight but exercise is more effective in reducing fat during the early phase of a weight-reducing program in healthy humans. J. Nutr. Biochem., 2003, 14(9), 541-9.

[21] Verdaet, D.; Dendale, P.; De-Bacquer, D.; Delanghe, J.; Block, P.; De-Backer, G. Association between leisure time physical activity and markers of chronic inflammation related to coronary heart disease. Atherosclerosis, 2004, 176(2), 303-10.

[22] Ignarro, L.J.; Balestrieri, M.L.; Napoli, C. Nutrition, physical activity, and cardiovascular disease: an update. Cardiovasc. Res., 2007, 73(2), 326-40.

[23] Smith, J.K.; Dykes, R.; Douglas, J.E.; Krishnaswamy, G.; Berk, S. Long-term exercise and atherogenic activity of blood mononuclear cells in persons at risk of developing ischemic heart disease. JAMA, 1999, 281(18), 1722-7.

[24] Pitsavos, C.; Chrysohoou, C.; Panagiotakos, D.B.; Skoumas, J.; Zeimbekis, A.; Kokkinos, P.; Stefanadis, C.; Toutouzas, P.K. Association of leisure-time physical activity on inflammation markers (C-reactive protein, white cell blood count, serum amyloid A, and fibrinogen) in healthy subjects (from the ATTICA study). Am. J. Cardiol., 2003, 91(3), 368-70.

[25] Reuben, D.B.; Judd-Hamilton, L.; Harris, T.B.; Seeman, T.E. MacArthur_Studies_of_Successful_Aging. The associations between physical activity and inflammatory markers in highfunctioning older persons: MacArthur Studies of Successful Aging. J. Am. Geriatr. Soc. 2003, 51(8), 1125-30.

[26] Reinehr, T.; Stoffel-Wagner, B.; Roth, C.L.; Andler, W. Highsensitive C-reactive protein, tumor necrosis factor alpha, and cardiovascular risk factors before and after weight loss in obese children. Metabolism, 2005, 54(9), 1155-61.

(C) Nascimento et al.; Licensee Bentham Open.

This is an open access article licensed under the terms of the Creative Commons Attribution Non-Commercial License (http://creativecommons.org/licenses/by-nc/3.0/) which permits unrestricted, non-commercial use, distribution and reproduction in any medium, provided the work is properly cited. 